\title{
A tale of two embolisations
}

\author{
M van Wyk, MB ChB, FCRadDiag (SA); E Loggenberg, MB ChB, MMed RadD
}

Unit of Interventional Radiology, Department of Radiology, University of the Free State, Bloemfontein, South Africa

Corresponding author: M van Wyk (matthysvw@gmail.com)

Chronic pulmonary tuberculosis may present as massive haemoptysis. Haemoptysis usually originates from the bronchial artery but the pulmonary artery might be the culprit vessel in recurrent haemoptysis. We present a case where bronchial artery embolisation had to be augmented by pulmonary artery coil embolisation for a Rasmussen's aneurysm after recurrent haemoptysis. In cases where recurrent haemorrhage occurs, sources other than the bronchial artery should be considered.

S Afr J Rad 2013;17(3):68-69. DOI:10.7196/SAJR.704

Massive haemoptysis, defined as the expectoration of $300-600 \mathrm{ml}$ of blood per day, is a frequent complication of acute and chronic pulmonary tuberculosis (PTB) (caused by Mycobacterium tuberculosis). Owing to epidemic HIV/AIDS in the South African population, PTB has flourished. Invariably, owing to their limited pulmonary reserve, such patients are poor surgical candidates. ${ }^{[1]}$ Systemic arterial embolisation by particles or metallic coils is therefore the core management in many of these patients. ${ }^{[1]}$ The haemoptysis usually originates from the bronchial arteries but rupture of an underlying Rasmussen aneurysm might cause recurrent haemoptysis, sometimes with fatal consequences. ${ }^{[2]}$

\section{Case report}

A 49-year-old man presented with a week-long history of haemoptysis, haematemesis and melena stools. He complained about loss of weight, a chronic cough and night sweats. The patient was known, with sputumproven PTB and on the continuation phase of PTB treatment. He was clinically anaemic with a haemoglobin level of $6.3 \mathrm{~g} / \mathrm{dl}$, hypotensive with a blood pressure of $100 / 60 \mathrm{mmHg}$, and tachycardic with a pulse rate of 100 BPM. The $\mathrm{Hb}$ was restored after transfusion of packed cells. Chest $\mathrm{X}$-ray revealed active pulmonary $\mathrm{TB}$ with destruction and cavitation of the right lung.

Once the patient was optimally resuscitated, selective bronchial arteriography revealed abnormal contrast extravasation from the right bronchial artery. This was successfully embolised with $300 \mu \mathrm{m}$ polyvinyl alcohol (PVA) particles (Cook, Bloomington, USA). The haemoptysis ceased and the patient improved clinically.

One week later, the patient returned with a recurrent episode of massive haemoptysis. His $\mathrm{Hb}$ dropped to $3.3 \mathrm{~g} / \mathrm{dl}$. CT angiography of the chest revealed a $2 \mathrm{~cm}$ oval aneurysm of the right upper lobe posterior segment pulmonary artery.

Selective embolisation of the Rasmussen aneurysm pedicle with one $3 \mathrm{~mm} \times 5 \mathrm{~cm}$ and two $3 \mathrm{~mm} \times 4 \mathrm{~cm}$ helical coils (Cook, Bloomington, USA) via a 5 Fr catheter achieved complete exclusion of the aneurysm. The patient's haemoptysis resolved over the next day.

\section{Discussion}

Massive haemoptysis usually originates from the bronchial circulation (95\%) rather than the pulmonary circulation (5\%). ${ }^{[2]}$ Conservative management of massive haemoptysis has a $50-100 \%$ mortality rate..$^{[2,3]}$ Published mortality rates for elective surgery performed for massive haemoptysis are less than $20 \% \cdot{ }^{[4]}$ The mortality rate for emergency surgical control, however, escalates to about $40 \%{ }^{[4]}$

Pulmonary tuberculosis can affect the pulmonary arteries and veins, leading to vasculitis and thrombosis. ${ }^{[5]}$ The bronchial arteries may enlarge and hypertrophy. ${ }^{[5]}$ Rasmussen aneurysm is a rare complication (in $5 \%$ of autopsied patients with cavitatory tuberculosis ${ }^{[6]}$ ) caused by pulmonary artery wall weakening owing to the adjacent cavitatory tuberculosis. ${ }^{[5]}$ Granulation tissue replaces the adventitia and media, leading to gradual weakening of the arterial wall. ${ }^{[5]}$ The granulation tissue is replaced by fibrin, leading to thinning, pseudoaneurysm formation and eventual rupture. ${ }^{[6]}$ In a study by Sanyika et al., 38\% of patients who re-bled after successful bronchial artery embolisations for haemoptysis had Rasmussen aneurysms. ${ }^{[1]}$ Haemoptysis is usually the presenting symptom and may be fatal when substantial. ${ }^{[6]}$ Since 1973, the use of bronchial artery embolisation (BAE) has become customary management for recurrent and massive haemoptysis. ${ }^{[2]}$ Recurrent haemoptysis occurs in approximately $20 \%$ of patients. ${ }^{[1,7]}$

Multidetector computed tomography (MDCT) has established itself as a very useful tool in identifying the precise location of the source of bleeding. ${ }^{[8]}$ Additionally, it may assist in identifying signs suggesting that the pulmonary artery is the source of bleeding, such as the presence of a proximal cavity, visualisation of a pulmonary artery bordering a cavity and a Rasmussen aneurysm. ${ }^{[8]}$

The present case serves to emphasise the fact that, in cases of recurrent haemoptysis, complicating pulmonary $\mathrm{TB}$, a CT angiogram of the pulmonary vessels should be considered, to exclude aneurysms that might lead to catastrophic haemoptysis. It furthermore illustrates the complementary role that particle and coil embolisation has to play in management of massive haemoptysis.

\footnotetext{
1. Kim HY, Song K-S, Goo JM, Lee JS, Lee KS, Lim T-H. Thoracic sequelae and complications of tuberculosis. RadioGraphics 2001;21:839-860.

2. Santelli ED, Katz DS, Goldschmidt AM, Thomas HA. Embolization of multiple Rasmussen aneurysms as a treatment of hemoptysis. Radiology 1994;193:396-398.

3. Najarian E, Morris CS. Arterial embolization in the chest. J Thorac Imaging 1998;13:93-104. [http:// dx.doi.org/10.1097/00005382-199804000-00004]
} 


\section{PICTORIAL INTERLUDE}

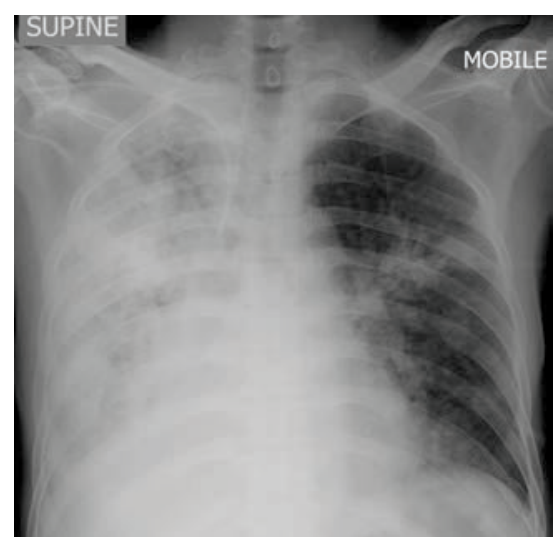

Fig. 1. Chest radiograph demonstrating cavitary pulmonary tuberculosis with marked destruction of the right lung.
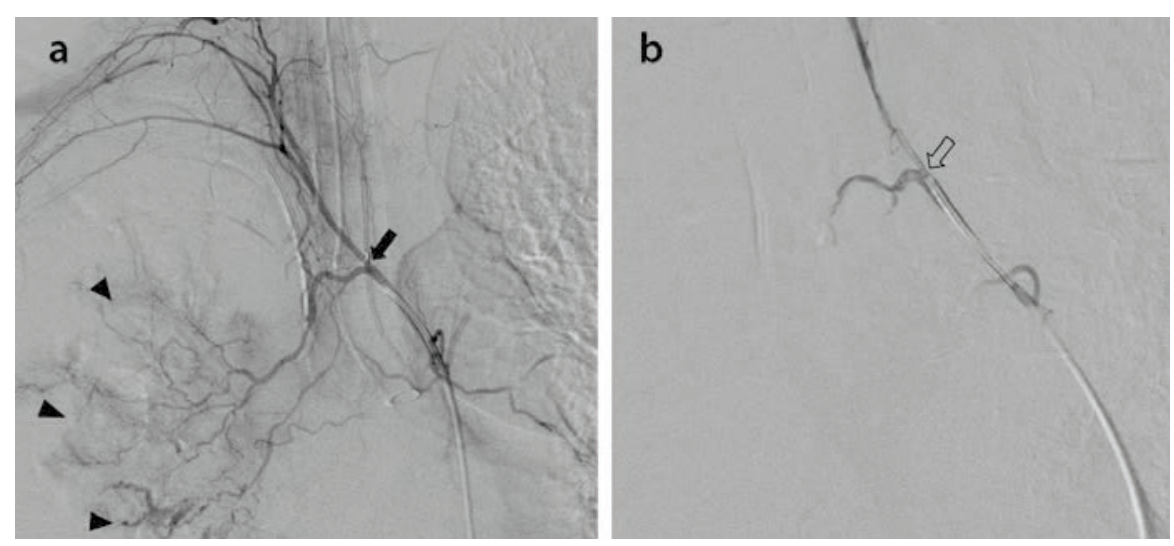

Fig. 2. (a) Selective right bronchial angiogram (arrow) demonstrates a large, hypervascular lesion in the right upper lobe (arrow heads). (b) Post-particle embolisation angiogram reveals complete obliteration of the hypervascular region.
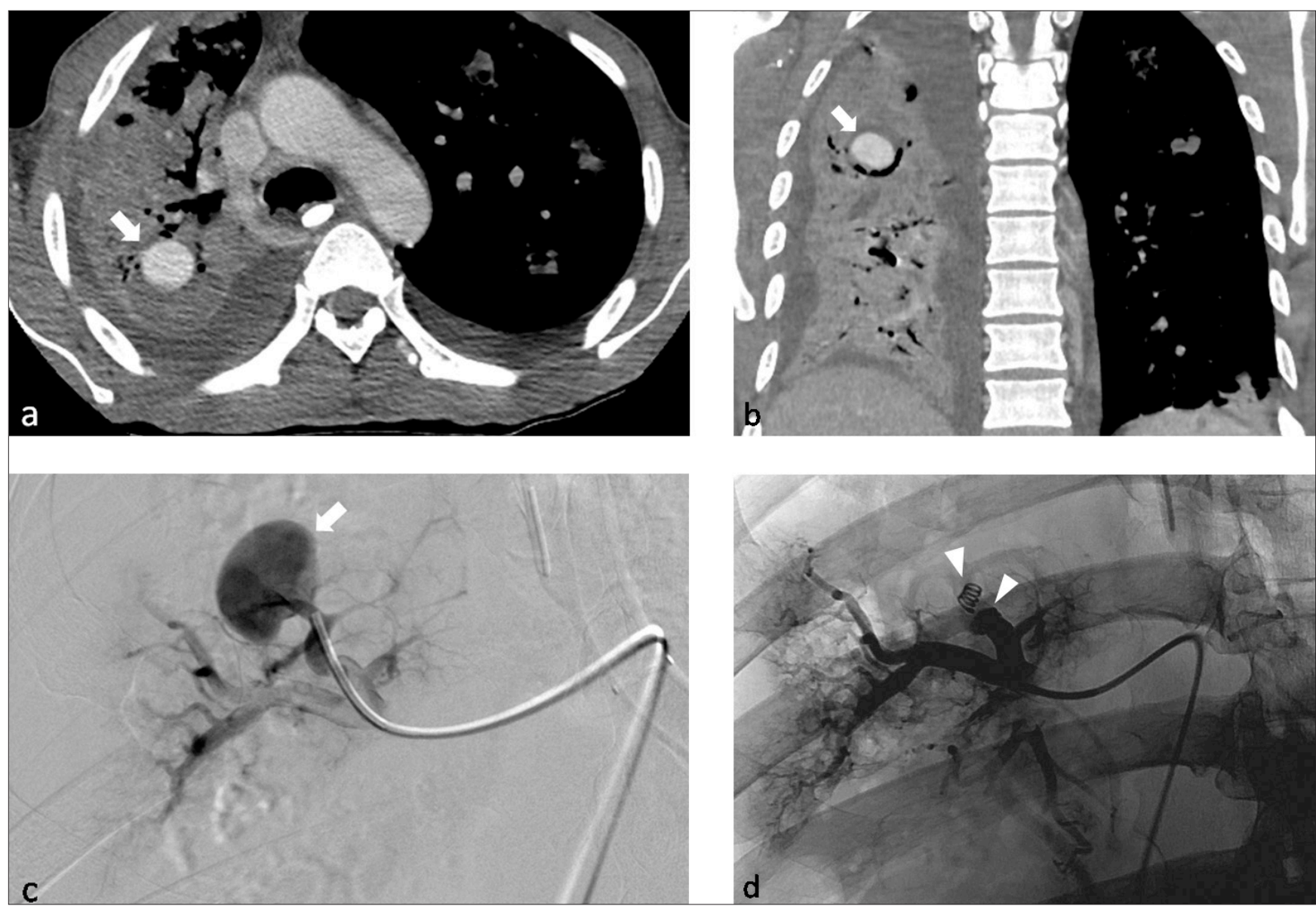

Fig. 3. (a) Axial contrast enhanced CT demonstrates filling of the Rasmussen aneurysm (arrow). The hyperdense ovoid structure in the oesophagus is a medicine tablet; (b) coronal reformation illustration of the aneurysm (arrow); (c) pulmonary angiogram demonstrates a Rasmussen aneurysm (arrow) in the right upper lobe posterior segment pulmonary artery; (d) post-embolisation pulmonary angiogram with occlusion of the vascular pedicle and exclusion of the Rasmussen aneurysm. The coils are indicated by the arrowheads.

4. Fernando HC, Stein M, Benfield JR, Link DP. Role of bronchial artery embolization in the management of hemoptysis. Arch Surg 1998;133:862-866. [http://dx.doi.org/10.1001/ archsurg.133.8.862]

5. Yoon W, Kim JK, Kim YH, Chung TW, Kang HK. Bronchial and nonbronchial systemic artery embolization for life threatening hemoptysis: a comprehensive review. RadioGraphics 2002;22:139-1409. [http://dx.doi. org $/ 10.1148 / \mathrm{rg} .226015180$

6. Sanyika C, Corr P, Royston D, Blyth DF. Pulmonary angiography and embolization for severe hemoptysis due to cavitatory pulmonary tuberculosis. Cardiovasc Intervent Radiol 1999;22:457-460. [http://dx.doi.org/10.1007/ s002709900432
7. Remy J, Lemaitre L, Lafitte J,, Vilian MO, Saint Michel J, Steenhouwer F. Massive hemoptysis of pulmonary origin: Diagnosis and treatment. AJR 1984;143:963-969. [http://dx.doi. org/10.2214/ajr.143.5.963]

8. Khalil A, Fartoukh M, Tassart M, Parrot A, Marsault C, Carette M-F. Role of MDCT in identification of the bleeding site and the vessels causing hemoptysis. AJR 2007;188:W117-W125. 